\title{
An unusual presentation of a ruptured abdominal aortic aneurysm
}

\author{
Adel Abdel Aziz $\cdot$ Nick Maskery
}

Published online: 18 September 2009

(C) Springer-Verlag 2009

\begin{abstract}
The diagnosis of ruptured abdominal aortic aneurysm (AAA) is often missed as a result of an unusual presentation, resulting in increased mortality. Here, we present an atypical presentation of ruptured AAA in a 72-year-old healthy male who had brief episode of syncope from which he fully recovered. A high index of clinical suspicion of ruptured AAA should be maintained in elderly patients presenting with history of syncope.
\end{abstract}

Keywords Ruptured abdominal aortic aneurysm . Left bundle branch block - Unusual presentation . Syncope

\section{Introduction}

Ruptured abdominal aortic aneurysm (AAA) is an important cause of unheralded death in people older then 65 years, making early recognition and intervention vital [1]. However, diagnosis is occasionally hampered by atypical presentation. We present this case report of one such patient who presented to our Emergency Department atypically and was haemodynamically stable.

\section{Case report}

A 72-year-old, ex-smoker with no previous past medical history presented to the Emergency Department with brief episode of syncope whilst on a caravan holiday. On

A. Abdel Aziz $(\bowtie) \cdot$ N. Maskery

Emergency Department, Southampton General Hospital, Tremona Road, Southampton, Hampshire SO16 6YD, UK e-mail: amabdelaziz@yahoo.com presentation to the Emergency Department he was well and asymptomatic. He was haemodynamically stable with normal blood glucose. His ECG revealed left bundle branch block (he had no chest pain at any time). There was no previous ECG available to compare. An ultrasound scan performed at the bedside revealed a 7.1-cm AAA. Whilst the ultrasound scan being performed, he became haemodynamically unstable. He was subsequently resuscitated and was taken to theatre for immediate repair within $25 \mathrm{~min}$. He had a good post-operative recovery and was discharged from the intensive care unit the next day to the vascular ward.

\section{Discussion}

The clinical manifestation of ruptured AAA can include low back pain, flank, abdominal or groin pain, which usually leads to hypovolaemic shock and altered mental status. The mortality of AAA rupture is high, $65-75 \%$ of patients die before they arrive at hospital and up to $90 \%$ die before they reach the operating room. The use of the ultrasound scan in Emergency Department is becoming increasingly popular in the UK. It is an extended bedside clinical examination, which can reflect on critical management decisions at early stage. It has been shown that Emergency Physicians can accurately perform aortic ultrasound scans with a relatively short training period [2]. Emergency ultrasound is a rapid, safe, non-invasive and repeatable test. It requires no contrast media and no special patient preparation. Additional benefits to clinicians such as surgeons and emergency physicians include continuing professional development and the satisfaction of broadened skills. Radiology Departments may benefit from decreased workload for out of hours in emergency imaging [3]. 


\section{Conclusion}

A limited ultrasound is a rapid screening test for the detection of AAA [4]. Emergency ultrasound examination has become almost a routine part of our clinical practice in Southampton Emergency Department. We are still on a learning curve. However, we have experienced significant benefits in patients care similar to the above-presented case.

Conflict of interest statement There is no conflict of interest related to the publication of this manuscript.

\section{References}

1. Brown LC, Powell JT (1999) Risk factors for aneurysm rupture in patients kept under ultrasound surveillance. UK small aneurysm trial participants. Ann Surg 230(3):289-296 (discussion 296-297)

2. Kuhn M, Bonnin RL, Davey MJ et al (2000) Emergency department ultrasound scanning for abdominal aortic aneurysm: accessible, accurate, and advantageous. Ann Emerg Med 36:219-223

3. Heller M, Verdille V (1992) Ultrasonography in emergency medicine. Emerg Med Clin North Am 10:27-44

4. Walker A, Brenchley J et al (2004) Ultrasound by emergency physicians to detect abdominal aortic aneurysms: a UK case series. Emerg Med J 21:257-259 